CLINICAL STUDY

\title{
Should genetic testing be performed in each patient with sporadic pheochromocytoma at presentation?
}

\author{
Pascal Pigny, Catherine Cardot-Bauters ${ }^{1}$, Christine Do $\mathrm{Cao}^{1}$, Marie Christine Vantyghem ${ }^{1}$, Bruno Carnaille ${ }^{2}$, \\ François Pattou ${ }^{2}$, Philippe Caron ${ }^{3}$, Jean-Louis Wemeau ${ }^{1}$ and Nicole Porchet \\ Laboratoire de Biochimie 'Hormonologie, Métabolisme-Nutrition, Oncologie', Centre de Biologie et Pathologie, CHRU de Lille, France, ${ }^{1}$ Service \\ d'Endocrinologie et Métabolismes, Clinique Marc Linquette, CHRU de Lille, 59037 France, ${ }^{2}$ Service de Chirurgie Endocrinienne, Hôpital Claude Huriez, \\ CHRU de Lille, France and ${ }^{3}$ Service d'Endocrinologie et Maladies Métaboliques, Hôpital Larrey, CHU de Toulouse, 31059 France \\ (Correspondence should be addressed to P Pigny; Email: p-pigny@chru-lille.fr)
}

\begin{abstract}
Background: According to previous studies, around 15\% of patients with an apparently sporadic pheochromocytoma and a negative family history had a hereditary disease. This high frequency together with the financial support provided to reference laboratories of molecular genetics by the French government led to a nearly systematic screening in each patient with a pheochromocytoma. Objective: To check the efficiency of systematic genetic screening in patients with apparently sporadic pheochromocytoma, by analysing the 6 years experience of a multidisciplinary team in this field. Methods: One hundred patients with a pheochromocytoma-only phenotype and no family history were included. Patients with extra-adrenal tumours were excluded. Prevalence of hereditary forms was determined and analyzed according to age at onset, sex. Cost of the genetic analysis was calculated. Results: A germline mutation in one of the five susceptibility genes (VHL, RET, SDHD, SDHC, SDHB) was identified in eight patients $(8 \%)$ with an age of onset between 13 and 57 years. Among them, six had a bilateral pheochromocytoma and only two had a unilateral tumour. If the guidelines for genetic screening were age of onset less than 50 or bilateral pheochromocytoma, no patients with a hereditary tumour would be missed and a $24 \%$ cost reduction would be achieved.

Conclusions: According to these data, a genetic predisposition test for hereditary pheochromocytoma seems not recommended in patients with a unilateral adrenal tumour diagnosed after 50 in the absence of familial, clinical, biological or imaging features for a familial disease.
\end{abstract}

European Journal of Endocrinology $160227-231$

\section{Introduction}

Pheochromocytomas are rare catecholamine-producing tumours that arise from chromaffin cells of the adrenal medulla. They are closely linked to paragangliomas that develop from others extra-adrenal neural crest derived cells such as parasympathetic or sympathetic paraganglia (1). However, pheochromocytomas are more commonly recognized because clinicians often focus on adrenal glands as the main source of catecholamine hypersecretion.

Until 1999, only 10\% of all pheochromocytomas were considered as hereditary tumours, and were part of multiple tumour syndromes such as multiple endocrine neoplasia type 2 (due to a germline mutation of the RET proto-oncogene), von Hippel-Lindau disease (VHL tumour-suppressor gene) or neurofibromatosis type 1 (NF1 tumour-suppressor gene) that are inherited on an autosomal-dominant mode. In the early 2000s, new susceptibility genes for hereditary pheochromocytomas and/or paragangliomas were discovered. These genes named SDHD, SDHB and SDHC encode three of the four protein subunits of the succinate dehydrogenase (SDH) also known as mitochondrial complex II (2). Therefore, at least six susceptibility genes for familial pheochromocytomas are now known (a number that could still expand in the next future (3)). The rapid transfer of these research data from the bench to the bedside has allowed re-evaluation of the true prevalence of hereditary forms of these adrenal tumours. Thus, in 2002, Prof Neumann from Freiburg University first demonstrated, by performing genetic testing in a cohort of 271 patients who presented apparently sporadic pheochromocytoma (in fact 241 with isolated pheochromocytoma), that about $24 \%$ of the patients did harbour a germline mutation of RET, SDHD, VHL or SDHB thus revealing a hereditary syndrome (4). However, exclusion of patients with a positive family history would decrease the rate of hereditary forms to $15.5 \%$ (4). In 2003, we reported a similar prevalence (15\%) by studying simultaneously five susceptibility genes (the former and $S D H C$ ) in 13 index cases (5). At the same time in France, President J. Chirac implanted the 'Plan Cancer' whose aim number 22 was to improve the access to genetic testing by providing a 
financial support to a network of reference laboratories specialized in molecular oncogenetics through the country. These two factors (high prevalence and financial support) explain that, in the last 5 years, genetic predisposition testing was nearly systematically proposed and done in each patient with a non-syndromic pheochromocytoma, at least in specialized medical centres. Our aim was to evaluate the efficiency of this systematic approach by analysing our 6 years experience in the field of pheochromocytoma molecular genetics. We were especially interested in the sensitivity and cost of systematic genetic testing. To get accurate guidelines, we decided to focus on patients with only pheochromocytoma and not to merge them with patients with functional paraganglioma, as frequently done in the literature (6).

\section{Research design and patients}

\section{Patients' selection}

In our medical centre, all patients with an apparently sporadic pheochromocytoma or paraganglioma (surgically removed and pathologically confirmed) had a consultation with either an endocrinologist with a specific skill in oncogenetics or a geneticist with a specific skill in endocrine tumours. Only patients who gave their signed informed consent for genetic testing were studied at the germline level. During the last 6 years (2002-2007) one hundred and forty index cases were recruited. Since our aim was to focus on patients with an apparently sporadic pheochromocytoma, we thus decided to exclude the 22 patients who suffered from an extra-adrenal abdominal paraganglioma first. Then we also excluded five patients who had a pheochromocytoma and a paraganglioma, eight patients with a pheochromocytoma and a positive familial history of adrenal tumour by inquiry and five patients with a pheochromocytoma and a positive familial history of von Hippel-Lindau disease by inquiry. Therefore, the sample analyzed here comprised of 100 patients (age: 13-95 years, male patients: 55, female patients: 45 ) who had either one or two non-syndromic pheochromocytoma without a positive familial history, without any other endocrine tumours, without any clinical signs for von Hippel-Lindau disease, MEN type 2 or neurofibromatosis type 1 . All patients had the clinical, biological and radiological investigations proposed in Table 1 . In this cohort of patients, pheochromocytoma was unilateral in 92 cases and bilateral in eight patients.

\section{Methods}

Genetic testing was proposed and performed once the patient gave his/her informed consent to do so either on his/her DNA or on those or her/his affected minor child. Genetic analysis consists in the search of germ line mutation of VHL, SDHB, SDHD, SDHC (all coding exons and intron-exon junctions are investigated) or RET (only exons 10, 11, 15 and 16 are studied) by PCR-sequencing on both strands of DNA, as previously described (5). For interpretation of genetic testing, a germline mutation was considered as pathogenic if i) it had already been described in the medical literature and/or ii) it was recorded in international genetic databases such as human gene mutation database or LOVD (7). By contrast, mutation was classified as not clearly pathogenic (nucleotide variant) if the available data were not convincing (new mutation without any functional study such as those affecting the NRF-2 cis-regulatory site of SDHB promoter) or if its status was controversial in the literature (for example VHL P25L variant (8)). For patients with bilateral pheochromocytoma who were mutation negative, a search for a deletion of VHL or SDHs was carried out by multiplex PCR or/and multiplex-ligation dependent probe assay (MLPA) respectively. The laboratory participates in the proficiency testing organized by the European Molecular genetics Quality Network-DNA sequencing scheme) and to the quality assurance testing organized by the French network of molecular biology laboratories working on endocrine tumours (GTE).

\section{Results}

A pathogenic germline mutation in one of the five susceptibility genes was found in eight patients $(8 \%)$, who revealed their pheochromocytoma between 13 and 57 years. There were four males and four females. As shown in Table 2, six patients had a bilateral pheochromocytoma due to a RET mutation (three unrelated cases), a VHL mutation (one case), a SDHD splice mutation (one case) or a partial deletion of $S D H B$

Table 1 Clinical features to be searched to identify a familial pheochromocytoma.

\begin{tabular}{|c|c|c|c|}
\hline Disease & Clinical investigation & Biological investigation & Imaging data \\
\hline$M E N-2$ & $\begin{array}{l}\text { Thyroid nodule } \\
\text { Renal lithiasis } \\
\text { Ganglioneuromatosis } \\
\text { Marfanoid habitus }\end{array}$ & $\begin{array}{l}\text { Basal calcitonin } \\
\text { Serum calcium, phosphate and PTH }\end{array}$ & \\
\hline$V H L$ & $\begin{array}{l}\text { Ophtalmoscopy } \\
\text { Retinal hemangioblastoma }\end{array}$ & & $\begin{array}{l}\text { Abdominal CT scan } \\
\text { Renal cysts or clear cell carcinomas } \\
\text { Pancreatic cysts or endocrine tumours }\end{array}$ \\
\hline$N F 1$ & $\begin{array}{l}\text { Café-au-lait spots } \\
\text { Neurofibromas } \\
\text { Iris hamartomas (Lisch nodules) }\end{array}$ & & \\
\hline
\end{tabular}


Table 2 Clinical data of the patients with a hereditary phaeochromocytoma.

\begin{tabular}{lccll}
\hline Patient number & Age at onset (years) & Sex (M/F) & Germ line mutation & Unilateral/bilateral phaeochromocytoma \\
\hline 1 & 13 & $\mathrm{M}$ & VHL Y156C & Bilateral pheochromocytoma \\
2 & 23 & $\mathrm{M}$ & RET C634R & Bilateral pheochromocytoma \\
3 & 35 & $\mathrm{M}$ & SDHD IVS2-1G $>\mathrm{T}$ & Unilateral phaechromocytoma \\
4 & 35 & $\mathrm{~F}$ & $R E T$ C634R & Bilateral pheochromocytoma \\
5 & 43 & $\mathrm{~F}$ & VHL Y156C & Unilateral pheochromocytoma \\
6 & 50 & $\mathrm{M}$ & SDHD IVS2-1G $>\mathrm{T}$ & Bilateral pheochromocytoma \\
7 & 57 & $\mathrm{~F}$ & $R E T$ C611R & Bilateral pheochromocytoma \\
8 & 46 & $\mathrm{~F}$ & SDHB exons 7-8 deletion & Bilateral pheochromocytoma \\
\hline
\end{tabular}

(one case). Two patients had a unilateral pheochromocytoma due to a SDHD or a VHL mutation. Moreover, the last two patients of this cohort with a bilateral pheochromocytoma who were mutation negative did not harbour a partial or complete deletion of $V H L$, SDHD, SDHB or SDHC. Eight patients $(8 \%)$, with a unilateral pheochromocytoma occurring between 24 and 70 years, had a non-clearly pathogenic nucleotide variant. In four cases, this variant was a $-7 \mathrm{G}>\mathrm{T}$ nucleotide change in the proximal NRF-2 cis-regulatory element of $S D H B$ promoter. Relationships between prevalence of the familial forms and age at discovery are shown in Table 3.

\section{Discussion}

As already shown in previous studies $(4,5)$ the younger the age at onset of pheochromocytoma, the higher the prevalence of hereditary tumours. On the contrary, the prevalence strongly decreases when age at onset is above 20 and becomes null when the age at discovery is strictly higher than 60. Sample size of the subgroups was, however, too small to perform a statistical analysis. Based on these prevalence data, if we had decided not to perform genetic testing in cases with an age of discovery strictly above 50 , we would have lost one patient with a hereditary tumour (sensitivity $=87.5 \%$ ) but have saved 32 useless genetic tests. If the age limit was set at 40 , we would have lost four patients with a true familial tumour, and thus decreased the sensitivity to $50 \%$. It should also be stressed that six (75\%) out of the eight patients with a bilateral pheochromocytoma had a hereditary form instead of only two $(2.2 \%)$ out of the 92 ones with a unilateral tumour. Therefore, if the decision criteria for genetic testing were age at onset less than 50 or bilateral pheochromocytoma, no patient with a hereditary tumour would be missed.

In our cohort of patients, only $8 \%$ had a familial pheochromocytoma, a frequency strongly lower than the prevalence previously published in the 2002-2003 $(4,5)$. This number is indeed also lower than the $12.7 \%$ prevalence recently reported by the European network for the study of adrenal tumours that broads together 545 patients with an apparently sporadic pheochro-mocytoma or a functional paraganglioma (6). However, our data fit well with those recently published by the French National Cancer Institute (INCa) in its 2002-2006 survey on oncogenetics activity (9). Indeed, in this survey, on the 314 index cases who had a germline analysis of $V H L$ and $S D H B$, and therefore probably harboured a pheochromocytoma, only $7 \%$ had a germline mutation.

\section{Who should benefit from genetic testing?}

Since 2002, the guidelines for genetic testing in patients with an apparently sporadic pheochromocytoma have been discussed and reviewed by two main studies. The first one coordinated by R.F Gagel from the USA (10), suggests performance of a genetic predisposition test to familial pheochromocytoma if $i)$ the tumour is discovered before 20 years, ii) the pheochromocytoma is bilateral or iii) in case of an extra-adrenal pheochromocytoma with catecholamine synthesis. Based on the data reported here, one can notice that all these clinical situations are characterized by a high prevalence of familial forms varying from between 33 and 75\%. More importantly, these American authors considered as useless or not justified the performance of genetic testing if age at diagnosis is above 50 years, when the prevalence is deeply lower: only $3 \%$ in our cohort of patients, $1.3 \%$ according to the analysis of Gagel and coworkers (10). Between 20 and 50 years, genetic testing is considered as optional, notably if the patient had no clinical signs in favour of a syndromic pheochromocytoma. In contrast, the European practice still considered that genetic testing should be systematic in every patient with either a pheochromocytoma or a paraganglioma (6). These authors recommend to select the genes to be screened

Table 3 Influence of the age at presentation on the prevalence of hereditary pheochromocytomas.

\begin{tabular}{llllllll}
\hline Age of onset (years) & $\mathbf{0 - 1 0}$ & $\mathbf{1 1 - 2 0}$ & $\mathbf{2 1 - 3 0}$ & $\mathbf{3 1 - 4 0}$ & $\mathbf{4 1 - 5 0}$ & $\mathbf{5 1 - 6 0}$ & $\mathbf{2 6 1}$ \\
\hline Patients number & 0 & 3 & 12 & 23 & 29 & 12 & 21 \\
Patients with a germline mutation & & 1 & 1 & 2 & 3 & 1 \\
Prevalence of hereditary pheos (\%) & & 33.3 & 8.3 & 8.7 & 10.3 & 8.3 & 0 \\
\hline
\end{tabular}


according to the clinical presentation and suggested analysis of SDHB and VHL in first intention in these patients. Such a clinically target molecular analysis is also recommended by the experts attending the first international symposium on pheochromocytomas (11).

\section{The cost of the genetic test should also be considered}

Health has no price but is a cost to the whole society. In France, genetic predisposition tests to hereditary cancers are performed in reference laboratories and are not charged to the patients because those labs received a specific financial support from the French INCa. However, the cost of these tests could be evaluated using a method proposed by the French association of molecular geneticists (ANPGM). This expert panel proposes to calculate the cost of each genetic test by adding the individual costs of each technical step performed, for example DNA extraction plus PCR-sequencing of each exon on both DNA strands. According to this method, the cost for searching for a germline VHL mutation by nucleotide sequencing (three exons) is $\sim 235$ euros, for a RET mutation (affecting exon 10, 11, 15 or 16) 270 euros, and for a complete analysis of the $3 \mathrm{SDH}$ genes is 1250 euros. Therefore, the cost of a complete genetic analysis of these five susceptibility genes by nucleotide sequencing is around 1755 euros. This cost does not include the search of complex molecular events not detected by nucleotide sequencing such as deletion(s)/insertion(s) or gene rearrangements. As shown in Fig. 1, we performed a cost analysis to compare two algorithms, systematic genetic testing versus target genetic testing. The first algorithm (consultation and systematic genetic testing) is those presently used in our medical centre. The second algorithm consists of testing only the patients who revealed their pheochromocytoma before 50 or/and had a bilateral tumour. This approach thus required a more detailed clinical survey to select the patients who will be eligible for genetic testing. Applying this algorithm would lead to a 42700 euros cost reduction ( $-24 \%)$. Moreover, using this approach, and based on our experience, no patients with a germ line mutation would be missed.

\section{Could we propose guidelines for performing genetic predisposition tests?}

Our aim is to propose guidelines that could be easily implanted in order to optimize our financial support. We believe that an intermediate statement could be proposed between the 'European' systematic screening and the 'American' restrictive screening. According to us, a genetic predisposition test for hereditary pheochromocytoma:

- should be performed in all patients with an age of onset less than 20 (prevalence of hereditary forms: $33.3 \%$ ) or with a bilateral pheochromocytoma (prevalence: $75 \%$ );

- is strongly recommended in patients with an age of onset less than 50 (cumulative prevalence: $10.4 \%$ );

- is not recommended in patients with a unilateral

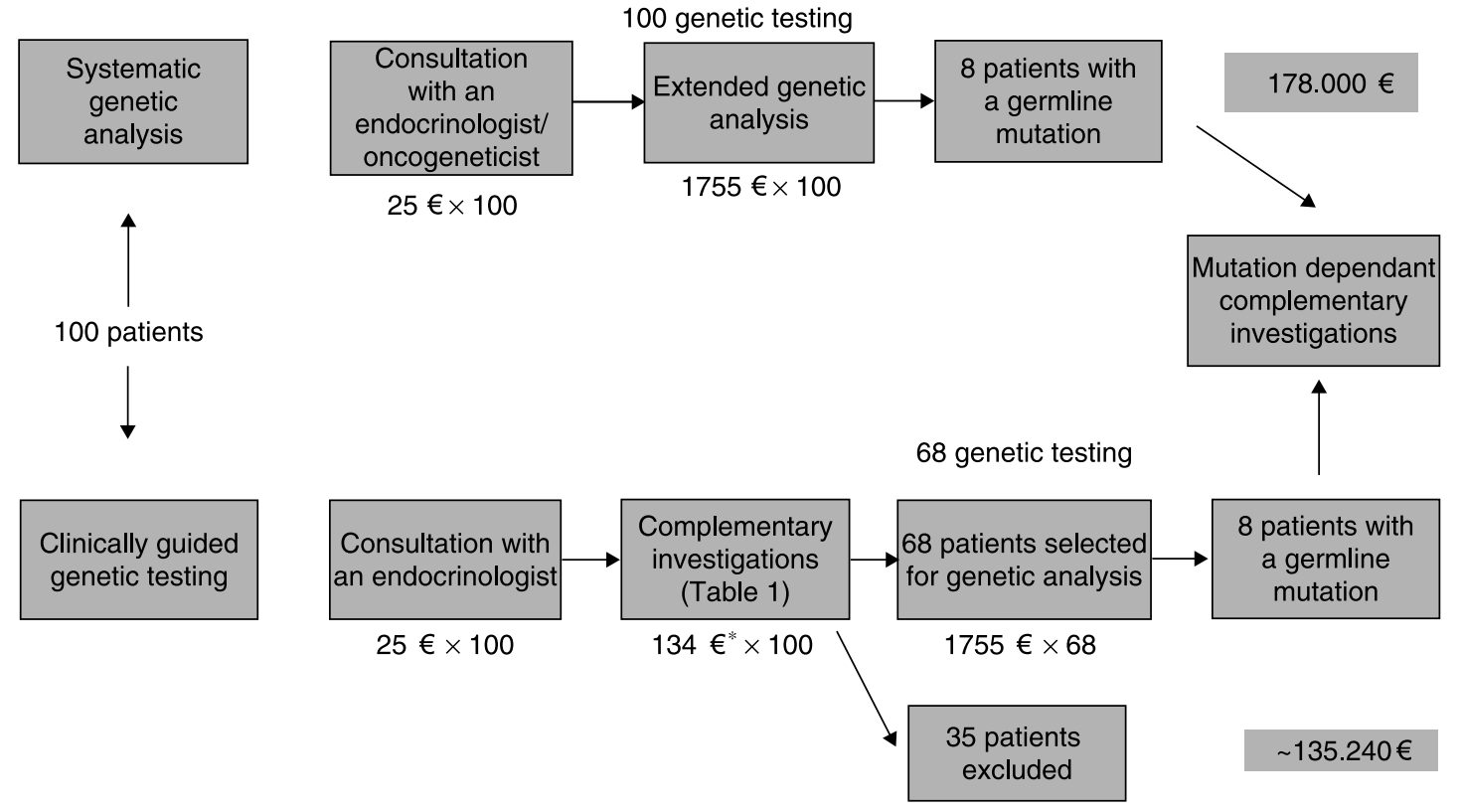

Figure 1 Cost analysis of the two algorithms, systematic genetic analysis (upper part) and clinically guided genetic analysis (lower part) meaning that only patients with an age of onset less than 50 or a bilateral pheochromocytoma will be eligible for genetic testing. For easier calculations, the costs were calculated for a sample of 100 patients. The clinically guided genetic testing would lead to a 42.700 euros cost reduction for 100 patients attending the outpatient visit. ${ }^{*}$ Cost of abdominal CT-scan excluded. 
pheochromocytoma diagnosed after 50 for whom neither the family history nor the clinical, biological or imaging data (performed as recommended in Table 3 and in (12)) are in favour of a familial disease. The occurrence of one new event during the patient's follow-up (that is systematic in our medical centre for every patient with a pheochromocytoma) would lead to reconsideration of the necessity of genetic testing.

In conclusion, genetic testing may help to predict for a patient the risk of multifocal tumours and/or malignancy, and to identify at risk relatives early. Nevertheless, we believe that the best strategy in terms of efficiency and cost has to modulate the use of genetic testing by clinical experience.

\section{Declaration of interest}

The contributing authors declare that there is no conflict of interest that could be perceived as prejudicing the impartiality of the research reported.

\section{Funding}

This research did not receive any specific grant from any funding agency in the public, commercial or not-for-profit sector.

\section{Acknowledgements}

We thank the following clinicians for their support and for providing clinical information: Drs O Verier-Mine, V Degros, M Ladsous (CH Valenciennes), Y Reznik, M Joubert (CHU Caen), R Desailloud (CHU Amiens), R Leroy, C Siame \& J P Cappoen (Lille).

\section{References}

1 Lewis CE \& Yeh MW. Molecular Genetics and Metabolism 200894 271-282.

2 Dahia PL. Evolving concepts in pheochromocytoma and paraganglioma. Current Opinion in Oncology 200618 1-8.
3 Schlisio S, Kenchappa RS, Vredeveld LCW, George RE, Stewart R, Greulich H, Shahriari K, Nguyen NV, Pigny P, Dahia PL, Pomeroy SL, Maris JM, Look T, Meyerson M, Peeper DS, Carter BD \& Kaelin WG. The kinesin KIFB acts downstream from EgIN3 to induce apoptosis and is a potential 1p36 tumor suppressor. Genes and Development 200822 884-893.

4 Neumann HP, Bausch B, McWhinney SR, Bender B, Gimm O, Franke G, Schipper J, Klisch J, Altehoefer C, Erres K, Januszewic A \& Eng C. Germline mutations in nonsyndromic pheochromocytoma. New England Journal of Medicine 2002346 1459-1466.

5 Bauters C, Vantyghem MC, Leteurtre E, Odou MF, Mouton C, Porchet N, Wemeau JL, Proye C \& Pigny P. Hereditary phaeochromocytomas and paragangliomas: a study of five susceptibility genes. Journal of Medical Genetics $2003 \mathbf{4 0}$ e75.

6 Gimenez-Roqueplo AP, Lehnert H, Mannelli M, Neumann H, Opocher G, Maher ER \& Plouin PF. Phaeochromocytoma, new genes and screening strategies. Clinical Endocrinology $2006 \mathbf{6 5}$ 699-705.

7 Bayley JP, Deville P \& Taschner PE. The SDH mutation database: an online resource for succinate dehydrogenase sequence variants involved in pheochromocytoma, paraganglioma and mitochondrial complex II deficiency. BMC Medical Genetics 2005 639.

8 Patocs A, Gergics P, Balogh K, Toth M, Fazakas F, Liko O \& Racz K. Ser80Ile mutation and a concurrent Pro25Leu variant of the VHL gene in an extented Hungarian von Hippel-Lindau family. BMC Medical Genetics 2008929.

9 Synthèse Nationale de L'évolution de L'activité D'oncogénétique 2003-2006. Institut National du Cancer, Mars 2008.

10 Jimenez C, Cote G, Arnold A \& Gagel RF. Should patients with apparently sporadic pheochromocytomas or paragangliomas be screened for hereditary syndromes? Journal of Clinical Endocrinology and Metabolism 200691 2851-2858.

11 Pacak K, Eisenhofer G, Ahlman H, Bornstein SR, Gimenez Roqueplo AP, Grossman AB, Kimura N, Mannelli M, McNicol AM \& Tischler AS. Pheochromocytoma: recommendations for clinical practice from the first International Symposium. Nature Clinical Practice. Endocrinology \& Metabolism 2007 3 92-102.

12 Pawlu C, Bausch B, Reisch N \& Neumann HPH. Genetic testing for pheochromocytoma-associated syndromes. Annales d'Endocrinologie $200566178-185$.

Received 17 November 2008

Accepted 19 November 2008 